\title{
INTERIOR AND BOUNDARY REGULARITY OF SOLUTIONS TO A PLASMA TYPE EQUATION
}

\author{
YING C. KWONG \\ (Communicated by Walter D. Littman)
}

\begin{abstract}
In this paper, we will consider a plasma type equation with homogeneous boundary condition and nonnegative initial data such that there is a finite extinction $T^{*}$. We will show that the solution is a positive classical solution in the interior of the parabolic cylinder and it decays to zero at the boundary at a certain explicit rate.
\end{abstract} lems

$$
\left\{\begin{array}{l}
\beta^{\prime}(u) u_{t}-\Delta u=0 \\
\left.u\right|_{\partial \Omega}=0 \\
u(0)=u_{0}, \quad u_{0} \geq 0, u_{0} \not \equiv 0
\end{array}\right.
$$

where $\beta$ satisfies

$$
\left\{\begin{array}{l}
\text { (i) } \beta \in C^{2}([0, \infty)), \quad \beta^{\prime}(s)>0 \text { for } s>0 \text { and } \beta(0)=\beta^{\prime}(0)=0, \\
\text { (ii) } \int_{0}^{1} \frac{d s}{\beta^{-1}(s)}<\infty,
\end{array}\right.
$$

where $\beta^{-1}$ is the inverse function of $\beta$. In this class of equations, there is the well-known plasma equation

$$
\left\{\begin{array}{l}
q u^{q-1} u_{t}-\Delta u=0, \quad q>2, \\
\left.u\right|_{\partial \Omega}=0, \quad u(0)=u_{0} \geq 0, u_{0} \neq \equiv 0 .
\end{array}\right.
$$

We first give a brief summary of the historical background of this class of problems for which the plasma equation is a prototype.

$1^{\circ}$. There is a finite extinction time, i.e. there exists $T^{*}>0$ such that $u\left(T^{*}\right) \equiv 0$. This extinction property has been studied by Gregorio Diaz-Ildefonso Diaz in [3] and Crandall and Benilan in [1].

$2^{\circ}$. As far as regularity is concerned, for $N=1,(1)$ has been studied by E. Sabanina. She proved the existence of positive classical solutions in [10]. For $N>1$, DiBenedetto in [4] and Paul Sacks in [11] proved the existence of a bounded weak solution which is continuous in $\Omega \times(0, \infty)$. Continuity up to the boundary $\partial \Omega$ has also been studied by DiBenedetto in [4]; however no explicit estimate on the modulus of continuity has been given in any of these cases.

$3^{\circ}$. It is a natural problem to investigate the asymptotic profile of the plasma equation as $T \rightarrow T^{*}$. This has been done in $[2,5]$.

Received by the editors October $5,1987$.

1980 Mathematics Subject Classification (1985 Revision). Primary 35B65, 35D10, 35K55.

Key words and phrases. Nonlinear degenerate diffusion equation, finite extinction time, regularity. 
The aim of this paper is to investigate both the interior and boundary regularity of the solution. As far as the interior regularity is concerned, it turns out that it is intimately related to the extinction time $T^{*}$. Indeed, we will show that the solution is positive in the interior of the parabolic cylinder $Q_{T^{*}}$, i.e. we are in the nondegenerate situation in the interior of $Q_{T^{*}}$ and hence we have the existence of a positive classical solution in $Q_{T^{*}}$ (Sabanina proved this result for the $N=1$ case). We will also prove that the solution decays to zero at $\partial \Omega$ at a certain explicit rate; this result is based on an explicit estimate derived in this paper. The virtue of this boundary estimate is the simplicity of the proof and the explicitness of its result.

II. Preliminaries. In this paper, we will adopt the same notations for the related functional spaces as in [8].

$1^{\circ}$. Weak solutions and the regularization $\left\{u_{n}^{\varepsilon}\right\}$ of $(1)$.

DEFINITION. Let the initial data $u_{0}$ be in $L^{\infty}(\Omega)$ and set $E=\left\{\psi \in \stackrel{\circ}{W}_{2}^{1.1}\left(Q_{T}\right) \mid\right.$ $\psi(x, T)=0$ a.e. on $\Omega\}$. An element $u$ of $\stackrel{\circ}{V}_{2}\left(Q_{T}\right)$ is a weak solution of (1) if $\beta(u) \in L^{2}\left(Q_{T}\right)$ and it satisfies

$$
\int_{Q_{T}} \int \beta(u) \psi_{t} d x d t-\int_{Q_{T}} \nabla u \cdot \nabla \psi d x d t+\int_{\Omega} \beta\left(u_{0}(x)\right) \psi(x, 0) d x=0
$$

for every $\psi \in E$.

It has been shown in [11] that if $u_{0} \in L^{\infty}(\Omega), u_{0}^{n} \in L_{0}^{\infty}(\Omega),\left\|u_{0}^{n}\right\|_{L^{\infty}(\Omega)} \leq$ $\left\|u_{0}\right\|_{L^{\infty}(\Omega)}$ and $u_{0}^{n} \rightarrow u_{0}$ in $L^{p}(\Omega)$ for every $1 \leq p<\infty$ as $n \rightarrow \infty$, then the standard regularization of $(1)$, namely

$$
(1, n, \varepsilon)\left\{\begin{array}{l}
\beta^{\prime}\left(u_{n}^{\varepsilon}\right) u_{n_{t}}^{\varepsilon}-\Delta u_{n}^{\varepsilon}=0,\left.\quad u_{n}^{\varepsilon}\right|_{\partial \Omega}=\varepsilon \\
u_{n}^{\varepsilon}(0)=u_{0}^{n}+\varepsilon
\end{array}\right.
$$

have solutions $u_{n}^{\varepsilon}$ which converge uniformly on compact subsets of $Q_{T}$ to the weak solution $u \in C\left(Q_{T}\right) \cap L^{\infty}\left(Q_{T}\right)$ of $(1)$ as $n \rightarrow \infty$ and $\varepsilon \rightarrow 0$ simultaneously.

$2^{\circ}$. Super and subsolutions of $(1)$.

DEFINITION. $\beta(\bar{u}) \in C\left((0, T] \mid L^{1}(\Omega)\right) \cap L^{\infty}\left(Q_{T}\right)$ is a supersolution of (1) if

$$
\int_{\Omega} \beta(u(T)) \rho(T) d x \geq \int_{\Omega} \beta\left(u_{0}\right) \rho(0) d x+\int_{Q_{T}}\left(\beta(u) \rho_{t}+u \Delta \rho\right) d x d t
$$

for every nonnegative $\rho \in C^{2,1}\left(\bar{Q}_{T}\right)$ with $\left.\rho\right|_{\partial \Omega}=0$. Subsolutions are defined analogously with $\geq$ replaced by $\leq$.

\section{Main results.}

THEOREM 1. Let $u(x, t)$ be the unique weak solution of (1) where $u_{0} \in L^{\infty}(\Omega)$, $u_{0} \not \equiv 0$ and $u_{0} \geq 0$. Then $u(x, t)$ is a positive classical solution of (1) in the cylinder $Q_{T^{*}}$ where $T^{*}$ is the extinction time. More precisely, $u(x, t)>0$ in $Q_{T^{*}}$, $u \in C^{2,1}\left(Q_{T}\right)$ and $u$ satisfies $\beta^{\prime}(u) u_{t}-\Delta u=0$.

THEOREM 2. Let $\beta$ satisfy (2) and $\partial \Omega$ be $C^{2}$, so that it satisfies the "exterior sphere condition" (i.e. for every $x_{0} \in \partial \Omega$, there exists a ball $B_{R}\left(x^{*}\right)$ of radius $R$ (uniformly on $x_{0}$ ) and center $x^{*}$, with $B_{R}\left(x^{*}\right) \cap \bar{\Omega}=\left\{x_{0}\right\}$ ). Then, if $u$ is $a$ weak solution of (1) with $u_{0} \in L^{\infty}(\Omega)$, for $T_{0}>0$ and $\left(x_{0}, T\right) \in \partial \Omega \times(0, \infty)$, $(x, T) \in \Omega \times(0, \infty)$, we have the estimate

$$
u(x, T) \leq \frac{2 N k\left\|u_{0}\right\|_{L^{\infty}(\Omega)} C^{2 N-1} d}{T R^{4 N}} \quad \text { where } d=\left|x-x_{0}\right|,
$$


$k$ is a constant depending on $\beta^{\prime}\left(\left\|u_{0}\right\|_{L^{\infty}(\Omega)}\right), N, R$ and $\Omega$ and $C$ satisfy $|x|<C$ for all $x \in \Omega$ ( $R$ being the radius in the exterior sphere condition).

\section{Proofs.}

PROOF OF THEOREM 1. $1^{\circ}$. This theorem is an immediate consequence of the following lemma. Let $\Omega_{T}$ denote $\left\{(x, t) \in Q_{T} \mid t=T\right\}$ and let $u(x, t)$ be the bounded continuous weak solution of (1). If $u(x, t)$ vanishes at $\left(x^{*}, T^{*}\right) \in Q_{T}$, then it vanishes everywhere on $\Omega_{T^{*}}$.

This lemma implies that $u$ is positive in $Q_{T^{*}}$. By standard results in [8], we can obtain uniform bounds on $u$ in $H^{2+\alpha, 1+\alpha / 2}\left(\overline{Q_{T}}\right)$ where $\alpha>0$ and $Q_{T} \subset \subset Q_{T}^{*}$. Hence, the degeneracy has been handled and we have a positive classical solution in $Q_{T^{*}}$.

PROOF OF THE LEMMA. $2^{\circ}$. This proof is a generalization of Sabanina's lemma in the $R^{1}$ case [10]. For simplicity we only provide a proof for $\Omega \subseteq R^{2}$; the $R^{N}$ case proceeds in exactly the same manner. We will argue by contradiction.

Indeed, let us assume the contrary. Then there exists a point $\left(x^{* *}, y^{*}, T^{*}\right)$ where $u$ does not vanish and consequently we can find a region $G$ in $Q_{T}$ as shown in the figure below such that $u(x, t)$ vanishes at $\left(x^{*}, y^{*}, T^{*}\right)$ but $u$ is greater than zero on $A_{3}$. (Here, without loss of generality, we assume $y^{*}=y^{* *}=0$ and $x^{* *}>x^{*}>0$ with $x^{*}=d$ and $x^{* *}=R$.) We let $(r, \sigma)$ denote polar coordinates and the pole of the coordinate system being located as in Figure I.

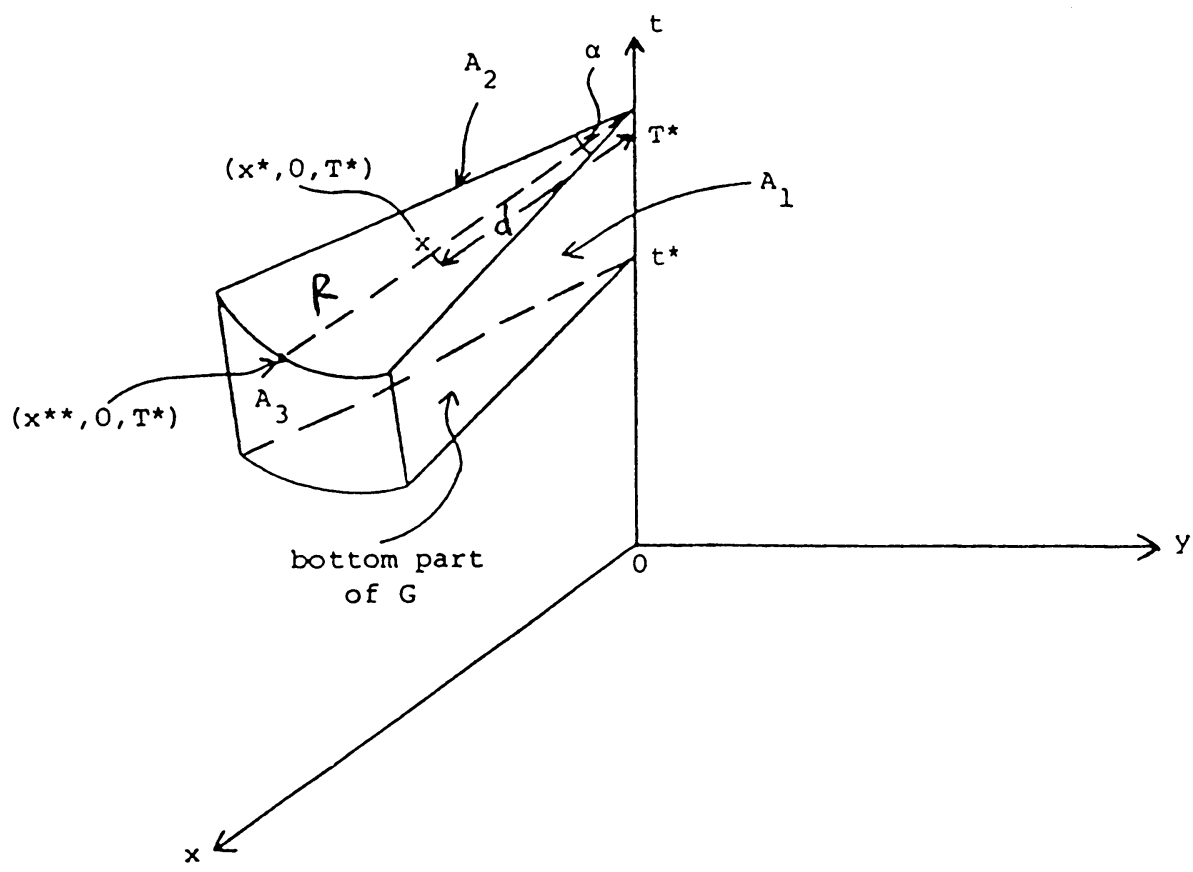

FIGURE I. The region $G$

We consider the following comparison function:

$$
w(x, y, t)=\left[m\left(t-t^{*}\right)+c\right] r^{\mu} \cos (\pi \sigma / 2 \alpha)-M \quad \text { where } \mu>\pi / 2 \alpha
$$


with $\mu>\pi / 2 \alpha$ and $0<c R^{\mu}<M<m\left(T^{*}-t^{*}\right) d^{\mu}$ where $m, M, c$ are positive constants so chosen that the inequality above holds. We define $L u=\beta^{\prime}(u) u_{t}-\Delta u$. Since

we have

$$
\Delta w=\frac{\partial^{2} w}{\partial r^{2}}+\frac{1}{r^{2}} \frac{\partial^{2} w}{\partial \sigma^{2}}+\frac{1}{r} \frac{\partial w}{\partial r}
$$

$$
\Delta \Lambda w=\Lambda \Delta w=\Lambda\left[m\left(t-t^{*}\right)+c\right]\left[\mu^{2}-(\pi / 2 \alpha)^{2}\right] r^{\mu-2} \cos (\pi \sigma / 2 \alpha)
$$

where $\Lambda>0$ is to be chosen later. Thus,

$$
\begin{aligned}
L(\Lambda w) & =\beta^{\prime}(\Lambda w) w_{t}-\Lambda \Delta w \\
= & \beta^{\prime}(\Lambda w) m \Lambda r^{\mu} \cos \left(\frac{\pi}{2 \alpha} \sigma\right)-\Lambda\left[m\left(t-t^{*}\right)+c\right]\left[\mu^{2}-\left(\frac{\pi}{2 \alpha}\right)^{2}\right] r^{\mu-2} \cos \left(\frac{\pi}{2 \alpha} \sigma\right) \\
= & \Lambda r^{\mu-2} \cos \left(\frac{\pi \sigma}{2 \alpha}\right)\left\{m r^{2} \beta^{\prime}(\Lambda w)-\left[m\left(t-t^{*}\right)+c\right]\left[\mu^{2}-\left(\frac{\pi}{2 \alpha}\right)^{2}\right]\right\} .
\end{aligned}
$$

Using the fact that $\beta^{\prime}(0)=0$ and $\beta^{\prime} \in C([0, \infty))$, we can choose $\Lambda$ sufficiently small so that $L(\Lambda w) \leq 0$ in $G,\left.\Lambda w\right|_{A_{1}}=\left.\Lambda w\right|_{A_{2}}=-\Lambda M<0$ and, at the bottom part of $G, w=c r^{\mu} \cos (\pi \sigma / 2 \alpha)-M \leq c R^{\mu}-M<0$.

Hence, by choosing $\Lambda$ sufficiently small, we may obtain $\left.\left(\Lambda w-u_{n}^{\varepsilon}\right)\right|_{A_{3}} \leq 0$. Thus in $G$, we have $L \Lambda w-L u_{n}^{\varepsilon} \leq 0$ with $\Lambda w-u_{n}^{\varepsilon} \leq 0$ along $A_{1}, A_{2}$ and $A_{3}$ and the bottom part of $G$. By invoking the maximum principle for the linear case (see [9] for those standard techniques), we conclude that $\Lambda w \leq u_{n}^{\varepsilon}(x, y, t)$ in $G$.

In particular, $0<\Lambda w\left(x^{*}, 0, T^{*}\right) \leq u_{n}^{\varepsilon}\left(x^{*}, 0, T^{*}\right)$. But $u_{n}^{\varepsilon}\left(x^{*}, 0, T^{*}\right) \rightarrow u\left(x^{*}, 0, T^{*}\right)$ $=0$ as $\varepsilon \rightarrow 0$ and $n \rightarrow \infty$. Hence, we have obtained a contradiction.

PROOF OF THEOREM 2. $1^{\circ}$. Again, we consider the regularization $\left\{u_{n}^{\varepsilon}\right\}$ defined by

$$
(1, n, \varepsilon)\left\{\begin{array}{l}
\beta^{\prime}\left(u_{n}^{\varepsilon}\right) u_{n_{t}}^{\varepsilon}-\Delta u_{n}^{\varepsilon}=0,\left.\quad u_{n}^{\varepsilon}\right|_{\partial \Omega}=\varepsilon \\
u_{n}^{\varepsilon}(0)=u_{0}^{n}+\varepsilon
\end{array}\right.
$$

Let $U_{n}^{\varepsilon}=u_{n}^{\varepsilon}-\varepsilon$ and $\beta_{\varepsilon}(s)=\beta(s+\varepsilon)$; then $U_{n}^{\varepsilon}$ satisfies

$$
\left\{\begin{array}{l}
\beta_{\varepsilon}^{\prime}\left(U_{n}^{\varepsilon}\right) U_{n_{t}}^{\varepsilon}-\Delta U_{n}^{\varepsilon}=0,\left.\quad U_{n}^{\varepsilon}\right|_{\partial \Omega}=0, \\
U_{n}^{\varepsilon}(0)=u_{0}^{n}
\end{array}\right.
$$

Multiplying by $\rho \in C^{2,1}\left(\overline{Q_{T}}\right),\left.\rho\right|_{\partial \Omega}=0, \rho \geq 0$ and integrating over $\Omega \times[0, T]$, yields

$$
\int_{Q_{T}} \beta_{\varepsilon}^{\prime}\left(U_{n}^{\varepsilon}\right) U_{n_{t}}^{\varepsilon} \rho-\int_{Q_{T}} \rho \Delta U_{n}^{\varepsilon}=0,
$$

which implies that $U_{n}^{\varepsilon}$ satisfies the corresponding integral equation

$$
\int_{\Omega} \beta_{\varepsilon}\left(U_{n}^{\varepsilon}(T)\right) \rho(T)-\int_{\Omega} \beta_{\varepsilon}\left(u_{0}^{n}\right) \rho(0)-\int_{Q_{T}} U_{n}^{\varepsilon} \Delta \rho+\beta_{\varepsilon}\left(U_{n}^{\varepsilon}\right) \rho_{t}=0 .
$$

$2^{\circ}$. Consider now any $x_{0} \in \partial \Omega$, and without loss of generality, assume that the center of the exterior sphere with respect to $x_{0}$ is at $(0, \ldots, 0)$. (Note that $|x|>R$ for $x \in \Omega$.) We construct the following function which turns out to a supersolution:

$$
f(x, t)=\left\|u_{0}\right\|_{L^{\infty}(\Omega)}\left[1-e^{-W(x) / t}\right]
$$

where $W(x)=k\left[1 / R^{2 N}-1 /|x|^{2 N}\right]$ and $k$ is to be determined later. We have

$$
f_{t}=-\left\|u_{0}\right\|_{L^{\infty}(\Omega)} e^{-W(x) / t} \frac{W(x)}{t^{2}}
$$


and

$$
\beta_{\varepsilon}^{\prime}(f) f_{t}=\frac{-\beta^{\prime}(f+\varepsilon)\left\|u_{0}\right\|_{L^{\infty}(\Omega)} W(x) e^{-W(x) / t}}{t^{2}}
$$

so that

$$
\beta^{\prime} \varepsilon(f) f_{t} \geq-\beta^{\prime}(f+\varepsilon)\left\|u_{0}\right\|_{L^{\infty}(\Omega)} k e^{-W(x) / t} / R^{2 N} t^{2} .
$$

Also,

$$
\nabla f=\left\|u_{0}\right\|_{L^{\infty}(\Omega)} e^{-W(x) / t} \frac{\nabla W}{t}
$$

and thus,

$$
\Delta f=\nabla \cdot \nabla f=-\left\|u_{0}\right\|_{L^{\infty}(\Omega)} e^{-W(x) / t} \frac{|\nabla W|^{2}}{t^{2}}+\left\|u_{0}\right\|_{L^{\infty}(\Omega)} e^{-W(x) / t} \frac{\Delta W}{t}
$$

where $|\nabla W(x)|^{2}=4 N^{2} k^{2} /|x|^{4 N+2}$. Finally,

$$
-\Delta W=-\left[\frac{2 N^{2} k}{|x|^{2 N+2}}-\frac{2 N k(N+2)}{|x|^{2 N+2}}\right]=\frac{4 N}{|x|^{2 N+2}} \geq 0 .
$$

Hence,

$$
-\Delta f \geq \frac{4 N^{2} k^{2}\left\|u_{0}\right\|_{L^{\infty}(\Omega)} e^{-W(x) / t}}{|x|^{4 N+2} t^{2}}
$$

which implies that

$$
\begin{aligned}
& \beta_{\varepsilon}^{\prime}(f) f_{t}-\Delta f=\frac{\left\|u_{0}\right\|_{L^{\infty}(\Omega)} e^{-W(x) / t}}{t^{2}}\left[\frac{4 N^{2} k^{2}}{|x|^{4 N+2}}-\beta^{\prime}(f+\varepsilon) W(x)\right] \\
& =\frac{\left\|u_{0}\right\|_{L^{\infty}(\Omega)} e^{-W(x) / t}}{t^{2}}\left[\frac{4 N^{2} k^{2}}{|x|^{4 N+2}}-\frac{k \beta^{\prime}(f+\varepsilon)}{R^{2 N}}\right] \\
& =\frac{k\left\|u_{0}\right\|_{L^{\infty}(\Omega)} e^{-W(x) / t}}{t^{2}}\left[\frac{4 N^{2} k}{|x|^{4 N+2}}-\frac{\beta^{\prime}\left(\left\|u_{0}\right\|_{L^{\infty}(\Omega)}\left(1-e^{-W(x) / t}\right)+\varepsilon\right)}{R^{2 N}}\right]
\end{aligned}
$$

and this will be $\geq 0$ provided we choose $k$ to be sufficiently large; we can do so because $\Omega$ is bounded. Here $k$ is of order $(|x| / R)^{2 N}|x|^{2 N+2}$.

$3^{\circ}$. It is now trivial to see that the $f$ so constructed is a supersolution of $U_{n}^{\varepsilon}$ (i.e. $f$ satisfies the same integral equation in $1^{\circ}$ with "=" replaced by " $\geq$ "). By the maximum principle (as proved and used in [5]), we have, subsolution $\leq$ supersolution. Thus, $U_{n}^{\varepsilon}(T) \leq f(T)$ so that

$$
u_{n}^{\varepsilon}(x, T) \leq f(x, T)+\varepsilon=\left\|u_{0}\right\|_{L^{\infty}(\Omega)}\left[1-e^{-W(x) / T}\right]+\varepsilon .
$$

$4^{\circ}$. Now let $\left(x_{0}, T\right) \in \partial \Omega \times(0, \infty), x \in \Omega$ and $d=\left|x-x_{0}\right|$; then $u_{n}^{\varepsilon}(x, T) \leq$ $\left\|u_{0}\right\|_{L^{\infty}(\Omega)}\left[1-e^{-W(x) / T}\right]+\varepsilon$. For $d$ small,

$$
\begin{aligned}
\left\|u_{0}\right\|_{L^{\infty}(\Omega)}\left[1-e^{-W(x) / T}\right]+\varepsilon & \approx\left\|u_{0}\right\|_{L^{\infty}(\Omega)}\left[1-\left(1-\frac{W(x)}{T}\right)\right]+\varepsilon \\
& =\frac{k\left\|u_{0}\right\|_{L^{\infty}(\Omega)}}{T}\left[\frac{1}{R^{2 N}}-\frac{1}{|x|^{2 N}}\right]+\varepsilon \\
& =\frac{k\left\|u_{0}\right\|_{L^{\infty}(\Omega)}}{T R^{2 N}|x|^{2 N}}\left[|x|^{2 N}-R^{2 N}\right]+\varepsilon .
\end{aligned}
$$


But,

$$
\begin{aligned}
|x|^{2 N}-R^{2 N} & =(|x|-R)\left(|x|^{2 N-1}+|x|^{2 N-2} R+\cdots+|x| R^{2 N-2}+R^{2 N-1}\right) \\
& \leq(R+d-R)(2 N) C^{2 N-1}=2 N C^{2 N-1} d
\end{aligned}
$$

where $R$ and $|x|$ for $x \in \Omega$, are both bounded by $C$. Now for $d$ small, then

$$
u_{n}^{\varepsilon}(x, T) \leq \frac{\left\|u_{0}\right\|_{L^{\infty}(\Omega)} 2 k N C^{2 N-1} d}{T R^{4 N}}+\varepsilon
$$

or alternatively,

$$
0 \leq u_{n}^{\varepsilon}(x, T)-\varepsilon \leq \frac{2 N k\left\|u_{0}\right\|_{L^{\infty}(\Omega)} C^{2 N-1} d}{T R^{4 N}},
$$

where $k$ is a constant dep nding on $\beta^{\prime}\left(\left\|u_{0}\right\|_{L^{\infty}(\Omega)}\right), N, R$ and $\Omega$. (One can easily see the dependence by going back to the process of choosing $k$ in step $2^{\circ}$.)

Recalling that $u_{n}^{\varepsilon}$ converges uniformly on compact subsets of $Q_{T}$ to $u$, we are done.

REMARKS. $1^{\circ}$. What we have obtained in the proof of Theorem 2 is not just a decay rate for $u(x, t)$ at the boundary $\partial \Omega$ but also a uniform decay rate on the regularization $\left\{u_{n}^{\varepsilon}\right\}$ at the boundary $\partial \Omega$.

$2^{\circ}$. It should be noted that we need $\beta \in C^{2}([0, \infty))$ to justify our computations with respect to the $u_{n}^{\varepsilon}$, i.e. to invoke the maximum principle and obtain the necessary regularity on the $u_{n}^{\varepsilon}$. In fact, it can also be assumed that $\beta$ is simply in $C^{\prime}([0, \infty))$, with $\beta^{\prime}(s)>0$ for $s>0$ and $\beta(0)=\beta^{\prime}(0)=0$; however in this case, one has to modify $\beta$ by replacing it by $\beta_{\varepsilon}$ where $\beta_{\varepsilon} \in C^{2}([0, \infty))$ and then let $\varepsilon \rightarrow 0$. In this case, although $u_{n}^{\varepsilon}$ still converges uniformly on compact subsets of $Q_{T}$, the regularity of $u$ will not be as good as in $C^{2,1}\left(Q_{T}\right)$ but somewhere in between $C\left(Q_{T}\right) \cap L^{\infty}\left(Q_{T}\right)$ and $C^{2,1}\left(Q_{T}\right)$. The optimal regularity in this case remains to be figured out.

$3^{\circ}$. It should be noted that throughout the whole paper, we have assumed $u_{0}$ to be in $L^{\infty}(\Omega)$. Due to the regularizing effect, for certain range of $q u(\cdot, t)$ becomes bounded once $T>0$ even if $u_{0}$ is implied in $L^{q-1}(\Omega)$. For this range of values of $q$, all the previous regularity results can be carried over for $u_{0}$ simply in $L^{q-1}(\Omega)$. For examples of this type of generalization, one may refer to the author's works in [5 and 6].

ACKNOWLEDGMENT. I would like to express my thanks to Professor Michael G. Crandall for his helpful discussions on this work; this paper is dedicated to Nancy and Janet who are very precious and special friends of mine. The author is also grateful for the helpful suggestions of the referee.

\section{REFERENCES}

1. P. Benilan and M. G. Crandall, The continuous dependence on $\phi$ of solutions of $u_{t}-\Delta \phi(u)=0$, Indiana Univ. Math. J. 30 (1981), 161-176.

2. James G. Berryman and Charles J. Holland, Stability of the separable solution for fast diffusion, Arch. Rational Mech. Anal. (1980).

3. Gregorio Diaz and Idlefonso Diaz, Finite extinction time for a class of non-linear parabolic equations, Comm. Partial Differential Equations 4 (1979), 1213-1231.

4. E. DiBenedetto, Continuity of weak solutions to a general porous medium equation, Indiana Univ. Math. J. 32 (1983), 83-118. 
5. Ying C. Kwong, The asymptotic behavior of the plasma equation with homogeneous Dirichlet boundary condition and non-negative initial data (in preparation).

6. __ Asymptotic behavior of plasma type equations with non-negative initial data and homogeneous Dirichlet boundary conditions (in preparation).

7. __ Asymptotic behavior of the plasma equation, TSR \#2727, Math. Research Center, Madison, Wisconsin, 1984.

8. O. A. Ladyzenskaja, V. A. Solonnikov and N. N. Ural'ceva, Linear and quasilinear equations of parabolic type, Math. Mono. 23, Amer. Math. Soc., Providence, R. I., 1968.

9. M. H. Protter and H. F. Weinberger, Maximum principles in differential equations, PrenticeHall, Englewood Cliffs, N. J., 1967.

10. E. S. Sabanina, A class of non-linear degenerate parabolic equations, Soviet Math. Dokl. 143 (1962), 495-498.

11. Paul Sacks, Continuity of solution of a singular parabolic equation, Nonlinear Anal. 7 (1983), 387-409.

Department of Mathematics, Northern Illinois University, DeKalb, IlliNOIS 60115 